\title{
A Shoulder Surfing Resistant Graphical Authentication System
}

\author{
B.Harish Goud , Indurthi Ravindra Kumar
}

Assistant Professor, Dept. of IT, JB INSTITUTE OF Engineering and Technology.

\section{Abstract:}

The likelihood of presenting passwords expanding to bear surfing assaults. Aggressors can watch straightforwardly or utilize outside account gadgets to gather client's accreditations. To conquer this issue, this undertaking proposed a novel confirmation framework PassMatrix in view of graphical passwords to oppose bear surfing assaults. Passmatrix offers no indication for aggressors; even they lead various camera based assaults.

\section{Keywords - Graphical Passwords, Authentication, Shoulder Surfing Attack.}

\section{INTRODUCTION}

Textual passwords have been the most generally utilized confirmation technique for a considerable length of time. Contained numbers and upper-and lower-case letters, printed passwords are viewed as sufficiently solid to oppose against animal power assaults. Be that as it may, a solid printed secret key is difficult to retain and remember [1]. Along these lines, clients have a tendency to pick passwords that are either short or from the word reference, instead of irregular alphanumeric strings. Surprisingly more terrible, it isn't an uncommon case that clients may utilize just a single username and secret word for different records [2]. As indicated by an article in Computer world, a security group at an extensive organization ran a system watchword saltine and shockingly split roughly $80 \%$ of the representatives' passwords inside 30 seconds [3]. Printed passwords are regularly unreliable because of the trouble of keeping up solid ones. Different graphical secret key validation plans [4], [5], [6], [7] were created to address the issues and shortcomings related with literary passwords. In light of a few examinations, for example, those in [8], [9], people have a superior capacity to retain pictures with long haul memory (LTM) than verbal portrayals. Picture based passwords were turned out to be less demanding to remember in a few client examines [10]. Thus, clients can set up a mind boggling verification watchword and are fit for remembering it after quite a while regardless of whether the memory isn't initiated occasionally. Nonetheless, the majority of these picture based passwords are defenseless against bear surfing assaults (SSAs). This kind of assault either utilizes coordinate perception, for example, viewing behind someone or applies video catching systems to get passwords, PINs, or other delicate individual data.

\section{RELEGATED WORK 2.1Existing System}

Keeping in mind the end goal to be more secure than the current Android design secret key with entropy 18:57 bits against savage power assaults, clients need to set two pass-pictures and utilize the graphical strategy to get the one-time login pointers. Like the greater part of other graphical secret key verification frameworks, PassMatrix is defenseless against irregular figure assaults in light of problem area investigating. Printed passwords have been 
the most generally utilized confirmation technique for a considerable length of time. Involved numbers and upper-and lower-case letters, literary passwords are viewed as sufficiently solid to oppose against animal power assaults. As indicated by an article in Computer world, a security group at an expansive organization ran a system secret word wafer and shockingly broke around $80 \%$ of the representatives' passwords inside 30 seconds. Printed passwords are regularly shaky because of the trouble of keeping up solid ones.

\subsection{Proposed System}

This development brings incredible accommodation yet additionally builds the likelihood of presenting passwords to bear surfing assaults. Assailants can watch straightforwardly or utilize outside chronicle gadgets to gather clients' accreditations. To conquer this issue, we proposed a novel validation framework PassMatrix, in light of graphical passwords to oppose bear surfing assaults. With a one-time substantial login pointer and circulative level and vertical bars covering the whole extent of passpictures, PassMatrix offers no indication for assailants to make sense of or limit the secret word even they lead various camerabased assaults. a considerable measure of research on secret word validation has been done in the writing. Among these proposed plans, this paper centers mostly around the graphical-based confirmation frameworks. To keep this paper compact, we will give a concise survey of the most related plans that were said in the past segment. The precision point of view centers around the fruitful login rates in the two sessions, including the training logins. The ease of use viewpoint is estimated by the measure of time clients spent in each PassMatrix stage.

\section{IMPLEMENTATION 3.1 IMAGE DESCRITIZATION MODULE:}

This module separates each picture into squares, from which clients would as the pass-square. A picture is isolated into a 7x11 network. The littler the picture is descritized, the bigger the secret word pick one space is. Consequently, in our execution, a division was set at 60 pixel interims in both level and vertical headings, since 60 pixel-square is the best size to precisely choose particular questions on touch screens.

\section{Flat AND VERTICAL AXIS CONTROL MODULE:}

There are two parchment bars: a flat bar with a grouping of letters and a vertical bar with a succession of numbers. This control module gives drag and excursion capacities to clients to control the two bars. Clients can throw either bar utilizing their finger to move one alphanumeric at once. They can likewise move a few checks at any given moment by dragging the bar for a separation. The bars are utilized to verifiably point the area of the clients pass-square.

\subsection{Login Indicator Generator Module:}

This module produces a login marker comprising of a few recognizable characters, for example, letter sets and numbers or visual materials, for example, hues and symbols for clients amid verification stage. In our usage, we utilized characters $A$ to $G$ and 1 to 11 for a $7 \times 11$ framework. The two letters and numbers are produced arbitrarily and in this manner an alternate login pointer is given each time the module is called.

\subsection{PASSWORD VERIFICATION MODULE:}

This module confirms the client secret key amid the validation stage. A pass-square acts like a secret key digit in the content based 
watchword framework. The client is confirmed just if the each pass-square in each pass-picture is effectively lined up with the login pointer

\subsection{COMMUNICATION MODULE:}

This module is responsible for all the data transmitted between the customer gadgets and the verification server. Any correspondence is secured by $\mathrm{SSL}$ (Secure Socket Layer) convention and subsequently, is protected from being listened stealthily and caught.

\section{EXPERIMENTAL RESULTS}

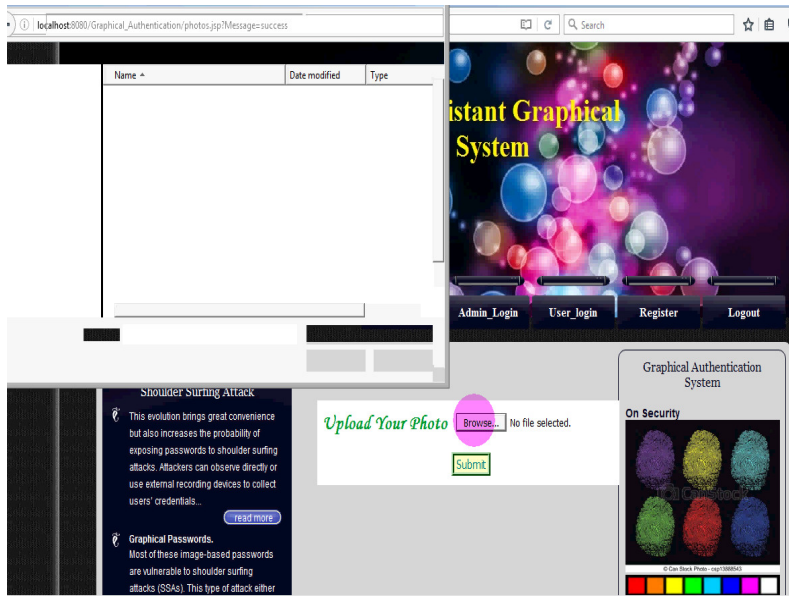

Fig 1 Choose file to upload

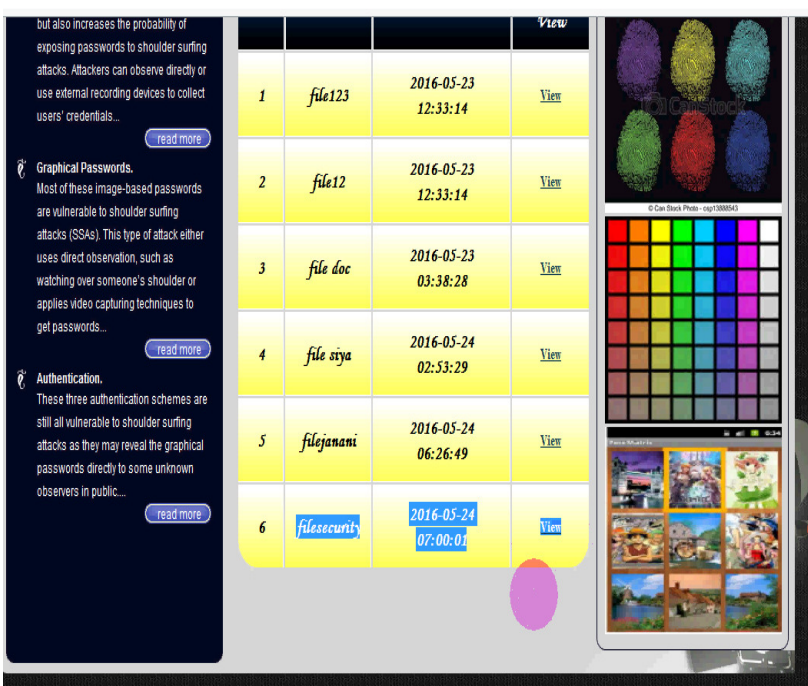

Fig 2 View files Page

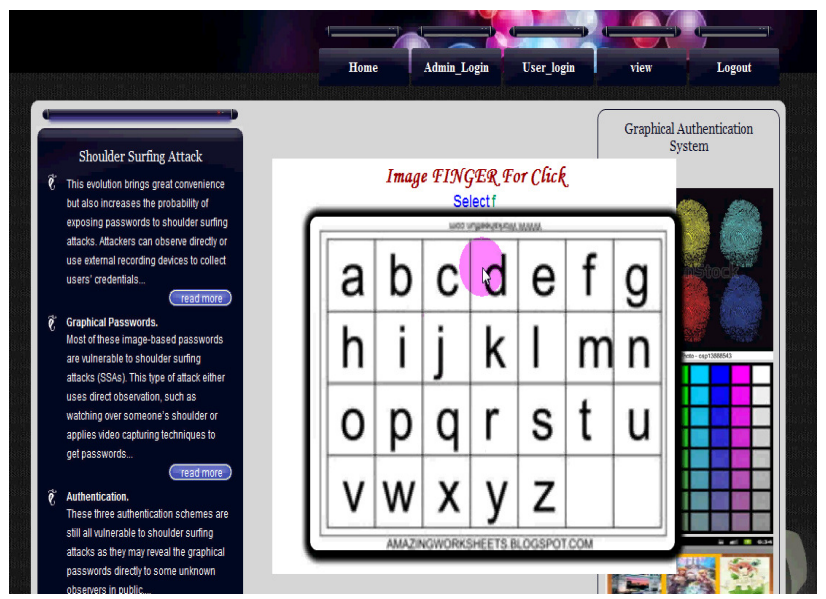

Fig 3 File upload Page

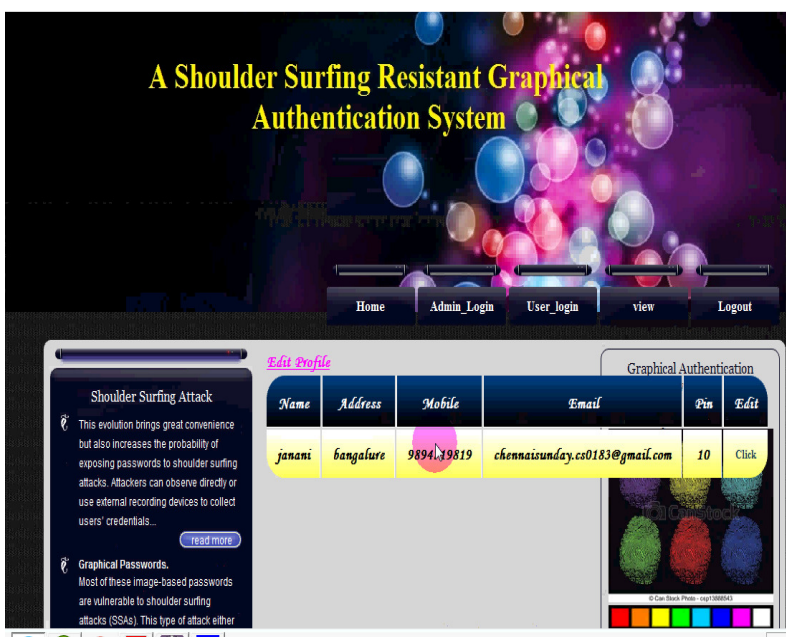

Fig 4Edit Profile

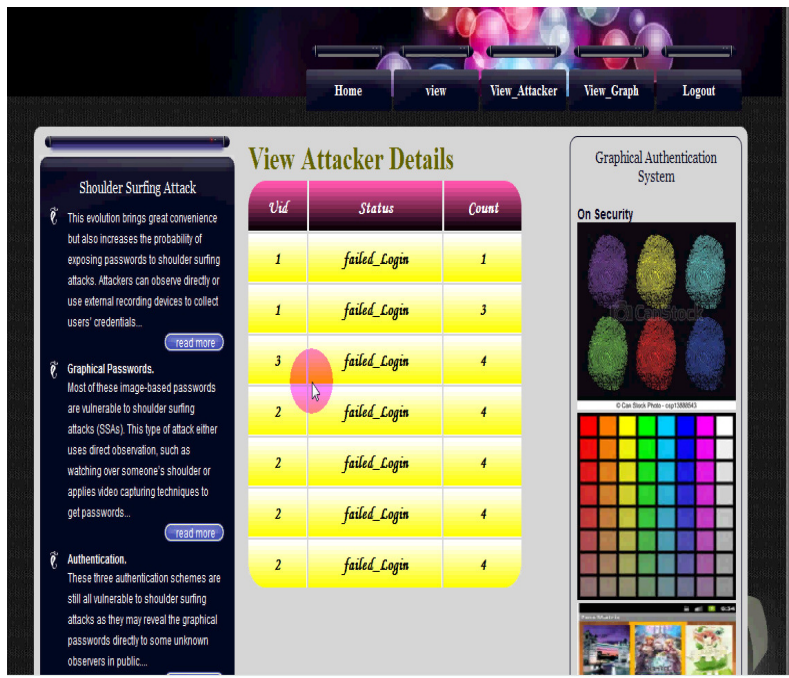

Fig 5View attacker details 


\section{CONCLUSION}

With the expanding pattern of web administrations and applications, clients can get to these applications whenever and anyplace with different gadgets. Keeping in mind the end goal to ensure clients' computerized property, validation is required each time they attempt to get to their own record and information. Utilizing conventional printed passwords or PIN strategy, clients need to type their passwords to validate themselves and hence these passwords can be uncovered effortlessly on the off chance that somebody looks over shoulder or uses video record To defeat this issue, we proposed a shoulder surfing safe verification framework in view of graphical passwords, named Pass Matrix. Utilizing a one-time login marker per picture, clients can bring up the area of their pass-square without specifically clicking or touching it, which is an activity defenseless against bear surfing assaults. In view of the outline of the flat and vertical bars that cover the whole pass-picture, it offers no hint for aggressors to limit the secret word space regardless of whether they have more than one login records of that record. Moreover, we actualized a Pass Matrix model on Android and completed client tests to assess the memorability and ease of use. The test result demonstrated that clients can sign into the framework with a normal of 1:64 tries (Median=1), and the Total Accuracy of all login trials is $93: 33 \%$ even two weeks after enrollment. The aggregate time devoured to sign into Pass Matrix with a normal of 3:2 pass-pictures is in the vicinity of 31:31 and $37: 11$ seconds and is viewed as worthy by $83: 33 \%$ of members in our client think about. The review information in the client think about likewise demonstrated that Pass Matrix is down to earth in reality. Mex Application is one of the valuable application in the present circumstance. This is the easy method to speak with the administrator. Worker cost assert work process turned into an early possibility for enablement as it could take out treatment of supporting cost bills and rather utilize the camera of Smartphone to catch the bill..

\section{REFERENCE}

[1] S. Sood, A. Sarje, and K. Singh, "Cryptanalysis of passwordauthentication schemes: Current status and key issues," in Methodsand Models in Computer Science, 2009. ICM2CS 2009. Proceeding ofInternational Conference on, Dec 2009, pp. 1-7.

[2] S. Gurav, L. Gawade, P. Rane, and N. Khochare, "Graphicalpassword authentication: Cloud securing scheme," in ElectronicSystems, Signal Processing and Computing Technologies (ICESC), 2014International Conference on, Jan 2014, pp. 479-483.

[3] K. Gilhooly, "Biometrics: Getting back to business," Computerworld,May, vol. 9, 2005.

[4] R. Dhamija and A. Perrig, "Deja vu: A user study using imagesfor authentication," in Proceedings of the 9th conference on USENIXSecurity Symposium-Volume 9. USENIX Association, 2000, pp. 4-4.

[5] "Realuser," http://www.realuser.com/.

[6] I. Jermyn, A. Mayer, F. Monrose, M. Reiter, and A. Rubin, "Thedesign and analysis of graphical passwords," in Proceedings of the8th conference on USENIX Security Symposium-Volume 8. USENIXAssociation, 1999, pp. 1-1.

[7] S. Wiedenbeck, J. Waters, J. Birget, A. Brodskiy, and N. Memon,"Passpoints: Design and longitudinal evaluation of a graphicalpassword system," International 
Journal of Human-Computer Studies,vol. 63, no. 1-2, pp. 102-127, 2005.

[8] A. Paivio, T. Rogers, and P. Smythe, "Why are pictures easier torecall than words?" Psychonomic Science, 1968.

[9] D. Nelson, U. Reed, and J. Walling, "Picture superiority effect,"Journal of Experimental Psychology: Human Learning and Memory,vol. 3, pp. 485-497, 1977.

[10] S. Brostoff and M. Sasse, "Are passfaces more usable than passwords?a field trial investigation," PEOPLE AND COMPUTERS,pp. 405-424, 2000. 